\title{
CONTINUOUS HOLDUP MEASUREMENTS WITH SILICON P-I-N PHOTODIODES
}

\section{Y-12}

NATIONAL SECURITY COMPLEX

MANAGED BY

BWXT Y-12, L.L.C.

FOR THE UNITED STATES

DEPARTMENT OF ENERGY

UCN-13672 (10-00)

\author{
J. A. Williams, D. E. Smith, M. J. Paulus \\ UT-Battelle, LLC \\ Oak Ridge National Laboratory \\ Oak Ridge, TN 37831, USA 865/576-8285 \\ Z. W. Bell, R. B. Oberer \\ BWXT Y-12, L.L.C. \\ Y-12 National Security Complex \\ Oak Ridge, TN 37831, USA 865/574-6120
}

May 2002 


\section{DISCLAIMER}

This report was prepared as an account of work sponsored by an agency of the United States Government. Neither the United States Government nor any agency thereof, nor any of their employees, makes any warranty, express or implied, or assumes any legal liability or responsibility for the accuracy, completeness, or usefulness of any in formation, apparatus, product, or process disclosed, or represents that its use would not infringe privately owned rights. Reference herein to any specific commercial product, process, or service by trade name, trademark, manufacturer, or otherwise, does not necessarily constitute or imply its endorsement, recommendation, or favoring by the United States Government or any agency thereof. The views and opinions of authors expressed herein do not necessarily state or reflect those of the United States Government or any agency thereof.

\section{COPYRIGHT NOTICE}

The submitted manuscript has been authored by a contractor of the U.S. Government under contract DE-AC05-00OR-22800. Accordingly, the U.S. Government retains a paid-up, nonexclusive, irrevocable, worldwide license to publish or reproduce the published form of this contribution, prepare derivative works, or distribute copies to the public, and perform publicly and display publicly, or allow others to do so, for U.S. Government purposes. 


\title{
CONTINUOUS HOLDUP MEASUREMENTS WITH SILICON P-I-N PHOTODIODES
}

\author{
J. A. Williams, D. E. Smith, M. J. Paulus \\ UT-Battelle, LLC \\ Oak Ridge National Laboratory \\ Oak Ridge, TN 37831, USA 865/576-8285 \\ Z. W. Bell, R. B. Oberer \\ BWXT Y-12, L.L.C. \\ Y-12 National Security Complex \\ Oak Ridge, TN 37831, USA 865/574-6120
}

\begin{abstract}
We report on the behavior of silicon P-I-N photodiodes used to perform holdup measurements on plumbing. These detectors differ from traditional scintillation detectors in that no high-voltage is required, no scintillator is used (gamma and X rays are converted directly by the diode), and they are considerably more compact. Although the small size of the diodes means they are not nearly as efficient as scintillation detectors, the diodes' size does mean that a detector module, including one or more diodes, pulse shaping electronics, analog-to-digital converter, embedded microprocessor, and digital interface can be realized in a package (excluding shielding) the size of a pocket calculator. This small size, coupled with only low-voltage power requirement, completely solidstate realization, and internal control functions allows these detectors to be strategically deployed on a permanent basis, thereby reducing or eliminating the need for manual holdup measurements. In this paper, we report on the measurement of gamma and $\mathrm{X}$ rays from ${ }^{235} \mathrm{U}$ and ${ }^{238} \mathrm{U}$ contained in steel pipe. We describe the features of the spectra, the electronics of the device and show how a network of them may be used to improve estimates of inventory in holdup.
\end{abstract}

\section{INTRODUCTION}

Manual measurement of uranium holdup in plumbing or ductwork is a labor-intensive and strenuous job. It may be performed in inhospitable environments (unheated, non-air conditioned, and/or contaminated, and/or confined spaces, for example), or require the handling of heavy, collimated detectors in awkward positions. The job typically requires a technician to hold a detector at a number of specified positions for a period of time and rapidly becomes tedious, and prone to error, 
while the technician becomes prone to injury. The job is expensive, costing a significant number of lost production days, and requiring the processing of personal protective equipment such as respirators, and anti-contamination clothing.

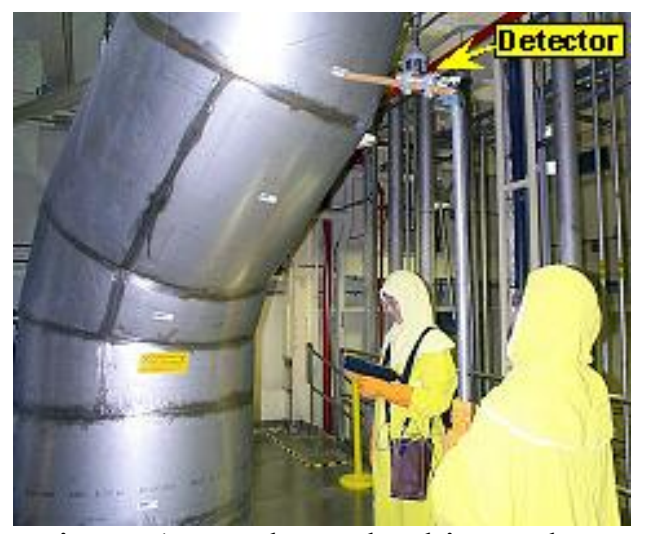

Figure 1, Workers checking a duct

Figure 1 shows two workers, wearing anti-contamination clothing, making a measurement. The worker in the foreground is holding a sodium iodide scintillation detector at the end of a boom. A meter stick is clamped to the boom and the worker has adjusted the position of the stick so that the detector is at the specified distance from a measurement point. The measurement points are indicated by the white stickers visible on the duct. The worker must consult a list of angles and distances to adjust the boom and meter stick for each measurement. Although this duct is easily accessible, plumbing, with multiple layers of closely laid pipe (sometimes much nearer the ceiling than the floor) presents an even more formidable challenge.

Therefore, we have developed detectors, based on silicon P-I-N diodes, that can be permanently mounted at measurement points, and which acquire and process spectroscopic information for transmission to a remote computer. These diodes, $200-500 \mu \mathrm{m}$ thick, directly convert low energy uranium gamma- and $x$-ray emissions and are sufficiently sensitive that a count rate of about $10 / \mathrm{sec} / \mathrm{mR} / \mathrm{hr}(200 \mu \mathrm{m}$ diode $)$ is observable above a threshold of $60 \mathrm{keV}$. Detectors based on these diodes have the advantages of lower weight, lower voltage, and lower cost when compared to traditional sodium iodide, but at the expense of significantly lower efficiency. However, the latter is not a major issue because, as will be seen below, spectra can be accumulated in under 20 minutes, and it is not required that a technician hold the detector during acquisition.

In the remainder of this paper, we describe measurements of uranium spectra with diodes 200, 300, and $500 \mu \mathrm{m}$ thick. A measurement was also performed with a "windowless" $300 \mu \mathrm{m}$ thick diode to test effects of the manufacturer's resin coating on the sensitivity of the detector and the quality of the spectrum. A description of the analog and digital electronics is given as well as the results of an MCNP simulation of the contribution to the spectrum from a uranium-containing pipe of uranium in nearby pipes.

\section{DETECTORS}

Since the goal of this effort is to develop a solid-state spectrometer, large-area diodes were selected. The radiation detectors used for these measurements were Hamamatsu silicon P-I-N photodiodes, as shown in Table 1. The diodes were operated at their maximum reverse bias voltage to insure 
maximum depletion. However, it was later learned that operation at $70 \%$ of maximum would have been sufficient to achieve over $90 \%$ depletion. Future work with these diodes will be at this lower voltage.

\begin{tabular}{|c|c|c|c|}
\hline Diode Type & Area $(\mathrm{mm} \times \mathrm{mm})$ & Thickness $(\mu \mathrm{m})[1]$ & Reverse Bias $(\mathrm{V})$ \\
\hline S3590-01 & $10 \times 10$ & 200 & 50 \\
\hline S3590-04 & $10 \times 10$ & 300 & 100 \\
\hline S3590-08 & $10 \times 10$ & 300 & 100 \\
\hline S3590-05 & $9 \times 9$ & 500 & 150 \\
\hline
\end{tabular}

Table 1, Diode characteristics

Chart 1 shows, on a logarithmic scale, the mean free path in silicon for photons undergoing coherent and incoherent scattering, and photoelectric absorption from 50 to $250 \mathrm{keV}[2]$. The total mean free path is also plotted.

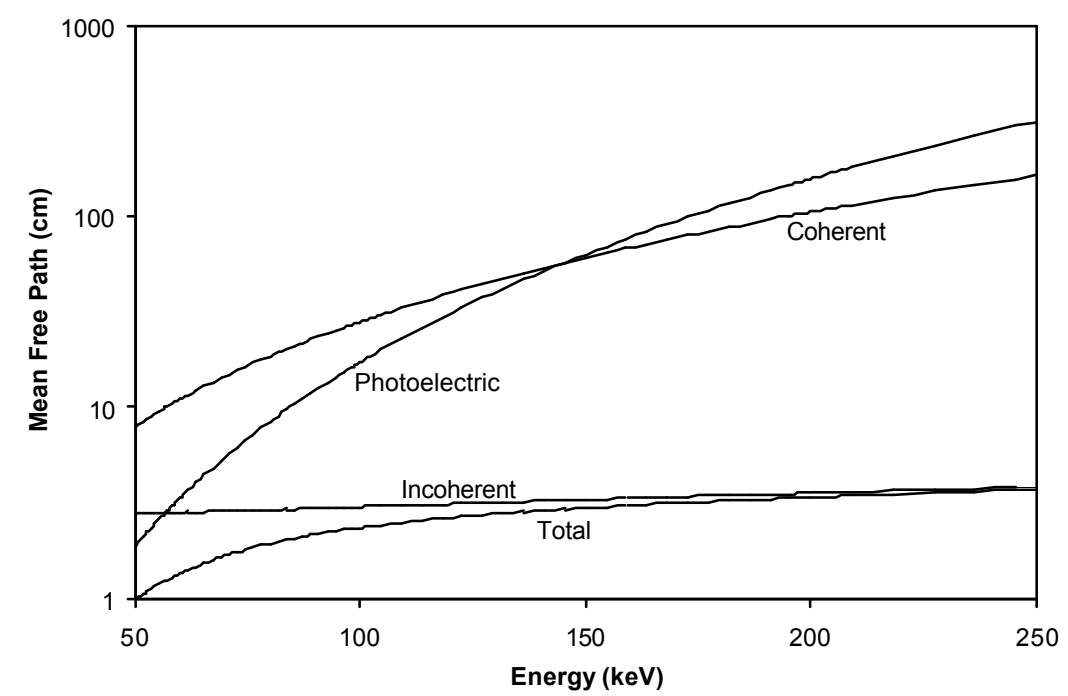

Chart 1, Mean free paths of photons for various absorption processes

Noting that smaller mean free path implies higher interaction cross section, the chart shows that the dominant process is incoherent scattering. The photoelectric mean free path (the parameter of interest for spectroscopy) is monotonically increasing between 50 and $200 \mathrm{keV}$ from about $2 \mathrm{~cm}$ to about $150 \mathrm{~cm}$, values far greater than the thickness of the diode. Using the chart and the thicknesses listed in Table 1, the absorption of 60,96 , and $186 \mathrm{keV}$ photons may be estimated. The results are shown in Table 2. The table takes into account the thickness of the resin window[3].

\begin{tabular}{|c|c|c|c|}
\hline Diode Type & $60 \mathrm{keV}$ & $96 \mathrm{keV}$ & $186 \mathrm{keV}$ \\
\hline S3590-01 & $0.60 \%$ & $0.13 \%$ & $0.016 \%$ \\
\hline S3590-04 & $0.90 \%$ & $0.20 \%$ & $0.024 \%$ \\
\hline S3590-08 & $0.90 \%$ & $0.20 \%$ & $0.024 \%$ \\
\hline S3590-05 & $1.49 \%$ & $0.33 \%$ & $0.040 \%$ \\
\hline
\end{tabular}

Table 2, Photoelectric absorption in diodes 
The table shows that theoretical photoelectric absorption scales according to the thickness of the depletion region; the lack of window in the S3590-04 has essentially no effect. It should be remembered that the S3590-05 has a surface area of only $81 \mathrm{~mm}^{2}$, while the others have $100 \mathrm{~mm}^{2}$. Consequently, although this diode should be $250 \%$ more efficient at the energies of interest, actual count rate should be only twice as great.

\section{ANALOG ELECTRONICS}

The analog circuit was constructed with surface mount components and fits on a circuit board is $8.75 \mathrm{~cm}$ long x $1.5 \mathrm{~cm}$, mounted inside a $9.2 \mathrm{~cm}$ long x $1.8 \mathrm{~cm}$ square aluminum tube, closed on each end by a copper plate. One of the copper plates is soldered to the circuit board.

Charge pulses caused by radiation incident on the reversed-biased diode were converted to a voltage pulses by a two-stage amplifier. The first stage employs a low-noise junction field-effect transistor $\left(<2 \mathrm{nv} / \mathrm{Hz}^{1 / 2}\right)$ in a high current gain, sub-unity voltage gain emitter-coupled circuit. The second stage is a common-base circuit using a bipolar transistor to provide high voltage gain with sub-unity current gain. Output signals from the second stage are connected directly to the inverting input of an operational amplifier. The operational amplifier feedback signal is connected to the input of the emitter-coupled amplifier circuit. An emitter-couple amplifier circuit is necessary because the capacitance coupled input signals from the silicon detectors are too small to drive the operational amplifier. Output signals from the operational amplifier are connected to a shaping circuit producing pulses approximately 7 microseconds wide. The bandwidth of three combined preamplifier and shaping circuits is approximately $40 \mathrm{kHz}$

The output of the shaping amplifer was fed to a peak detector and hold circuit shown in Figure 2. A pulse acquisition cycle begins with a positive-going reset pulse at the gate of Q2, discharging the voltage stored on $\mathrm{C} 1$. As long as the voltage at the non-inverting terminal of $\mathrm{U} 1$ is greater than the held voltage on $\mathrm{C} 1, \mathrm{D} 2$ conducts, and the base-emitter junction of Q1 is forward biased, causing the voltage on $\mathrm{C} 1$ and the output of $\mathrm{U} 2$ follow the input of U1. Diode D1 is reversed biased.

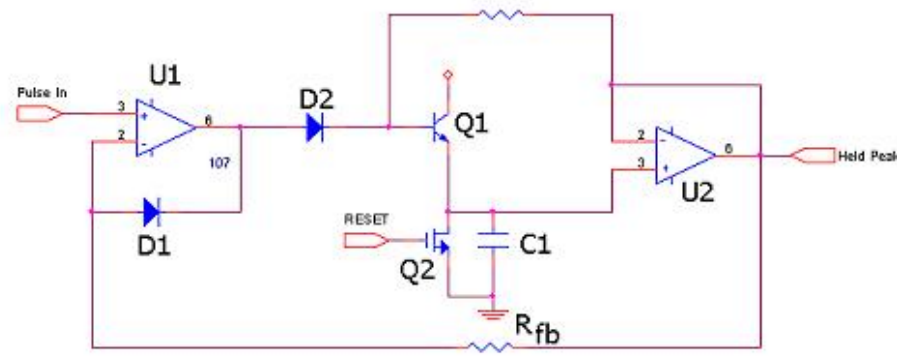

Figure 2, Peak detect and hold circuit[4]

After the pulse peak is reached and the input signal falls below the voltage on C1, D1 becomes forward biased because the voltage on $\mathrm{C} 1$ cannot change instantaneously. This causes a negative differential input voltage at U1, and a negative-going voltage at its output, driving the anode of D2 to one diode drop below the input signal. D2 becomes reverse biased because the base of Q1 (and the cathode of D2) is at a voltage no lower than of the voltage of C1 (which is already greater than the input voltage at U1). With D2 reverse biased, the feedback loop is broken, Q1 is biased off (because the resistor between the base of Q1 and the inverting terminal of U2 causes the base- 
emitter voltage to be zero), and U2 holds the output voltage at the peak. With Q1 off, the output voltage droops only because of U2's input bias currents.

\section{DIGITAL ELECTRONICS}

The integrated components in the digital circuit are low power surface mount parts with pin compatible derivatives operating at the $5.0 \mathrm{~V}$ logic level. The $5 \mathrm{~V}$ digital logic derivatives were chosen since the analog was designed around 5V logic. A block diagram of the main components of the digital subsection is shown in Figure 3.

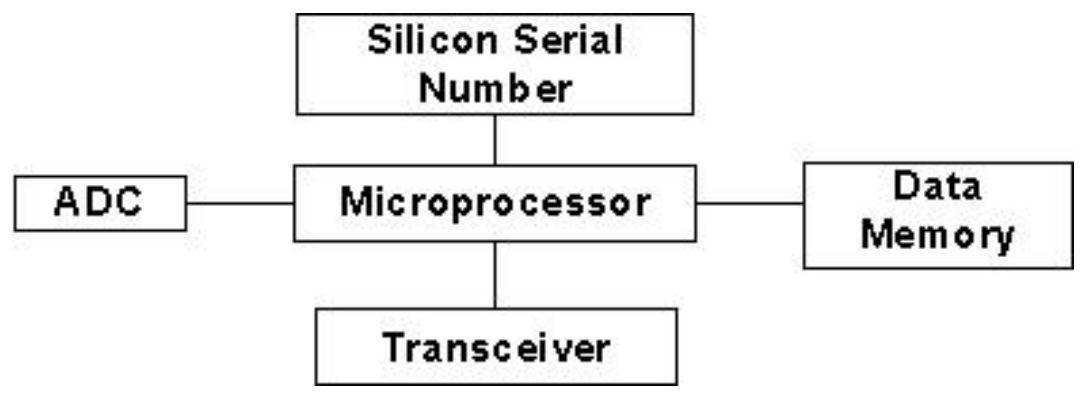

Figure 3, Digital subsection block diagram

The device is controlled by a Philips Semiconductor P89C51RD+ microprocessor having 64K bytes of code memory and $1 \mathrm{~K}$ byte of random-access memory (RAM). The microprocessor is clocked at $12 \mathrm{MHz}$. An additional 32K bytes of static RAM is provided by a Cypress CY62256 static RAM interfaced to the microprocessor through a 74VHC373 octal D-Type latch. A Maxim MAX813L microprocessor supervisory circuit controls the microprocessor's power-on reset and low-power reset. A Maxim MAX1242 ADC (10 bits), interfaced to the microprocessor through its serial peripheral interface (SPI), converts the signals from the analog circuit to digital values. A Maxim MAX483E RS-485 transceiver is used for the communication link to the host computer. A Dallas Semiconductor DS2401 silicon serial number integrated circuit (IC) is interfaced to the microcomputer by a 1-wire bus system. The silicon serial number provides unique identification for each device.

The device is initialized by a series of firmware routines when power is applied and communication between the host computer and the device is established after the initialization process is complete. The host computer and the device communicate via serial hardware protocol over a half-duplex RS485 line at baud rates up to 115,200 . On power-up, the spectrometer is initialized to send a command over the serial interface to the host to indicate its presence and availability for service. It then waits for commands from the host.

To start sampling, the microprocessor sends a reset pulse to the analog circuit to discharge the sample-and-hold. When the analog circuit detects a pulse exceeding a fixed threshold, a ready pulse is sent to the microprocessor to trigger the ADC to perform a conversion, sample the ADC, and increment the spectrum in memory. A reset pulse is then sent to the analog circuit indicating that the digital circuit is ready for another sample. The sampling process is continued until the programmed sampling time interval is complete or a limit condition (such as channel overflow) is sensed. The data is analyzed after each completion of a sampling time interval. 


\section{SPECTRA}

1000-second spectra from an 80 gram, $99 \%{ }^{235} \mathrm{U}$ source were obtained from each of the four types of diode and are shown in Chart 2. The photopeaks from the main emissions from ${ }^{235} \mathrm{U}$ are plainly visible, as is the Compton edge (at $78 \mathrm{keV}$ ) from the $186 \mathrm{keV}$ gamma ray. As expected, increasing the thickness of the diode increases the efficiency of detection. However, analysis of the $186 \mathrm{keV}$ photopeaks indicates that the count rates per unit area of diode are in the ratio of 1:2.5:3.24 (-01:04/-08:-05) while Table 2 predicts them to be in the ratio of 1:1.5:2.5. MCNP simulations of diode wafers irradiated by a parallel source of $186 \mathrm{keV}$ photons indicated that the ratios should be 1:1.9:3.8. The discrepancy between MCNP and Table 2 is understood to be due to the escape of photoelectrons (range of approximately $200 \mu \mathrm{m}$ in $\mathrm{Si}$ ), which was not taken into account in developing Table 2 .

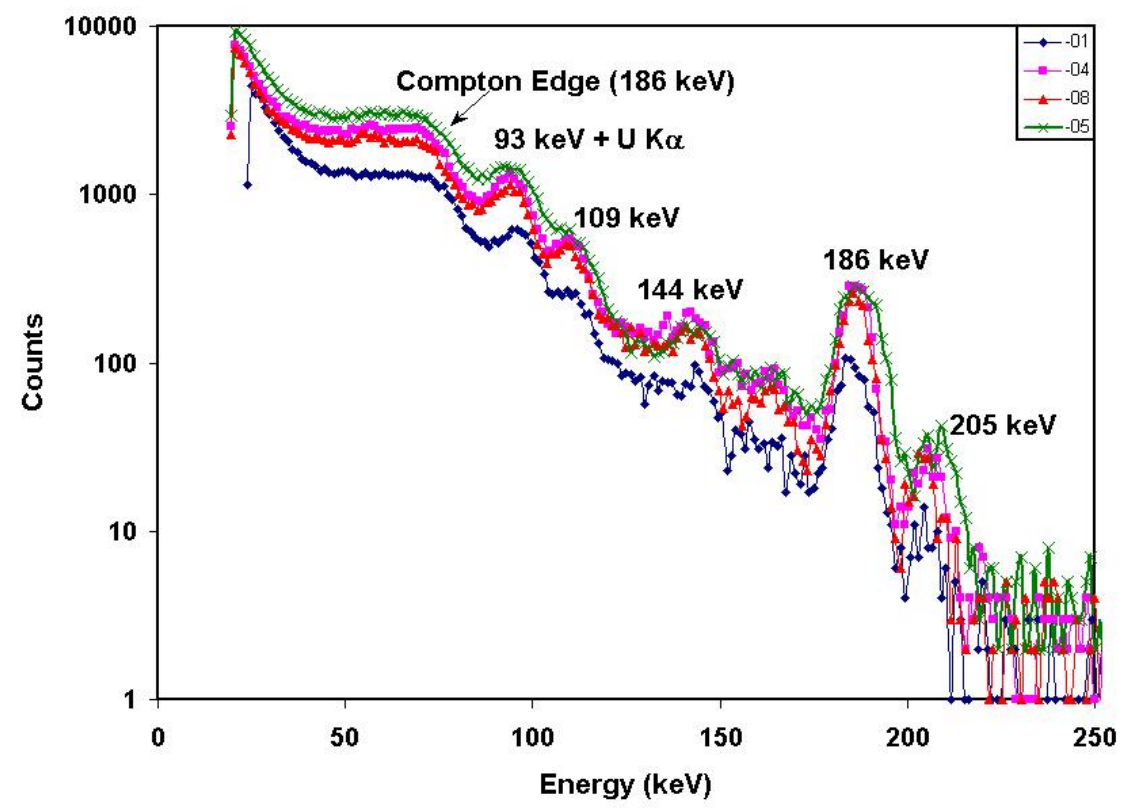

Chart 2, Spectra from the four types of diode (1000 seconds)

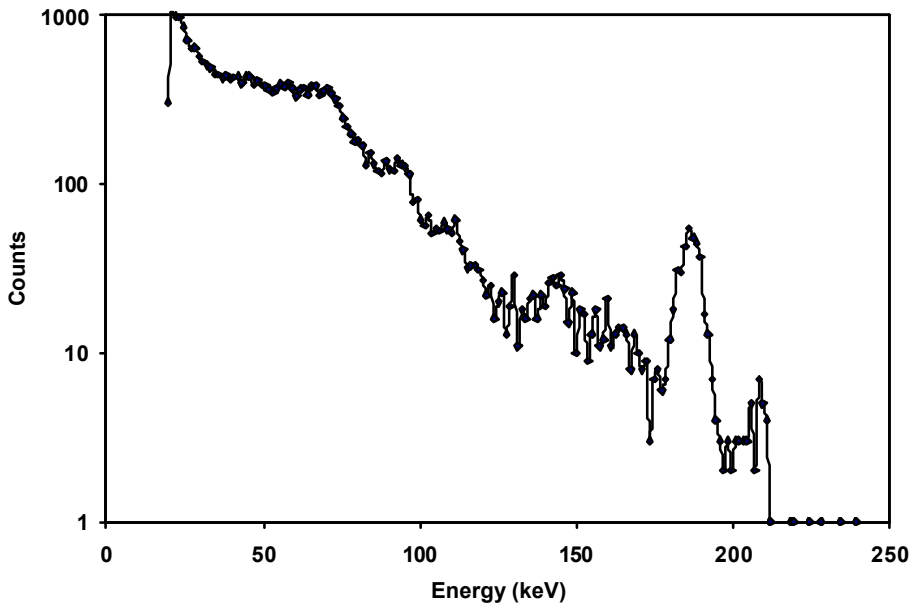

Chart 3, Uranium in a pipe (-05 diode, 1000 seconds) 
Chart 3 shows the spectrum obtained by a -05 diode $(500 \mu \mathrm{m})$ from 6 grams of powdered $99 \%{ }^{235} \mathrm{U}$ arranged along in a line along the wall of a schedule-40 steel pipe, against which was butted the detector (see Figure 4). Although the pipe severely attenuates the 93, and $109 \mathrm{keV}$ gamma rays, and x-rays, the intensity of the $186 \mathrm{keV}$ gamma ray is decreased only by a factor of about 5 and is easily discernible in the spectrum. Subsequent simulations (see below) suggest that the Compton continuum above $250 \mathrm{keV}$ from the high-energy ${ }^{238} \mathrm{U}$ emissions should be easily seen and that an estimate of enrichment should be derivable from these spectra.

\section{EFFECTS OF NEARBY SOURCES}

Figure 4 shows the MCNP model of the deployment of a -05 detector $1 \mathrm{~cm}$ below the outside wall of a 2-inch diameter schedule-40 steel pipe containing a point source at its bottom. A steel collimator surrounded the detector, and a second source was placed in an adjacent pipe. The effects of varying the thickness of the collimator wall from 0 (no collimator) to $2 \mathrm{~cm}$ were modeled. The purple area in Figure 4 is empty space, the green is the steel of the pipes, and the blue is the steel of the collimator. The horizontal line inside the collimator represents the detector.
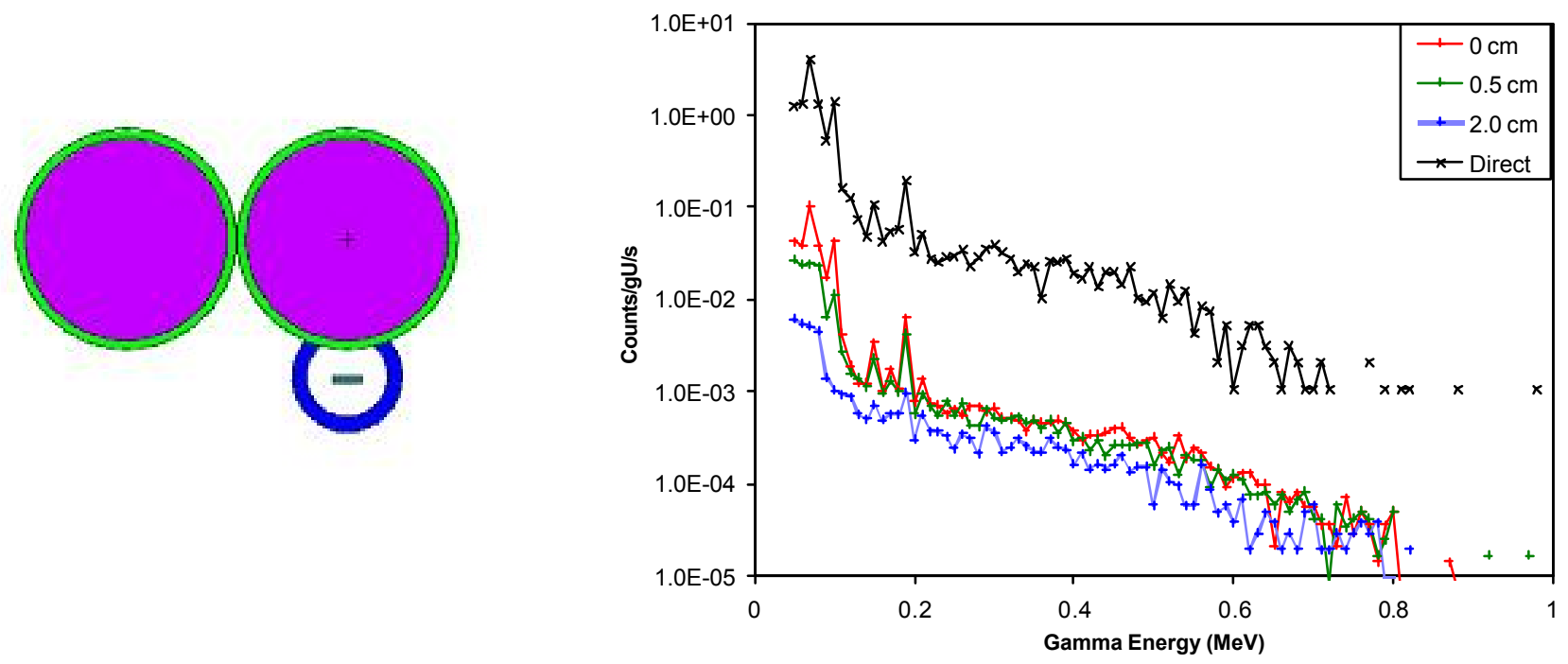

Figure 4, Geometry of MCNP simulation

Chart 4, Effects of collimator thickness

Chart 4 shows the effects of increasing thickness of the collimator; the data is displayed for a simulation of $15 \%{ }^{235} \mathrm{U}$ sources in both the main and adjacent pipes. This enrichment was chosen for display so that the features of the ${ }^{238} \mathrm{U}$ and ${ }^{235} \mathrm{U}$ components of the spectrum would be visible. The simulation does not take self-absorption into account; neither does it account for detector resolution. Note the logarithmic scale.

The main features of Chart 4 are the Compton edge from the $186 \mathrm{keV}$ photons, the Compton continuum from the $1001 \mathrm{keV}{ }^{238} \mathrm{U}$ photons, discrete lines from ${ }^{235} \mathrm{U}$ at $205,186,163$, and $144 \mathrm{keV}$, and a line at $63 \mathrm{keV}$ from ${ }^{234} \mathrm{Th}$. In addition, a cluster centered at $95 \mathrm{keV}$ contains contributions from $\mathrm{U}$ and $\mathrm{Th} \mathrm{K}_{\alpha} \mathrm{X}$-rays as well as ${ }^{234} \mathrm{Th}$ gamma rays. 
It is seen that the direct component is overwhelmingly dominant, even without collimation. This is a consequence of the small solid angle subtended by a $1-\mathrm{cm}$ detector seen obliquely from a source several centimeters distant. Although the insertion of $0.5 \mathrm{~cm}$ of steel provides noticeable attenuation below $100 \mathrm{keV}$, it has little effect on the $186 \mathrm{keV}$ emission, because of the small linear attenuation coefficient of steel at that energy. However, increasing the thickness to $2 \mathrm{~cm}$ eliminates all unwanted ${ }^{235} \mathrm{U}$ emissions. At $186 \mathrm{keV}$, the combination of geometry and shielding reduces the contribution from the left-hand pipe by a factor of approximately 175 . Thus, with only a small amount of shielding, the presence of a large nearby source can be ignored.

\section{DISCUSSION}

The data and simulations presented above demonstrate that a spectrometer based on a $500 \mu \mathrm{m}$-thick silicon P-I-N photodiode can provide information sufficient to recognize, distinguish, and quantify the ${ }^{235} \mathrm{U}$ and ${ }^{238} \mathrm{U}$ components of the spectrum. The spectrum contains both photopeaks from lowenergy emissions and Compton continuum from $1001 \mathrm{keV}{ }^{238} \mathrm{U}$ gamma rays. A useful spectrum can be accumulated in about 15 minutes, a time far too long for a manually operated device, but more than adequate for automatic operation in a stationary process.

Use of a multi-drop RS-485 communications protocol allows a single computer to monitor and control a large number of devices in a network. RS-485 lines may be 1000 meters in length, and can support dozens of clients. By polling each spectrometer, either on demand or on a schedule, current measurements of holdup can be up-loaded for trend analysis, or analysis of enrichment. The speed of measurement results in the accumulation of many measurements per day and permits statistical analysis for early prediction of problem accumulations.

Components for the spectrometer carry a cost of approximately $\$ 300.00$ for the detector, analog, and digital electronics, and case. Including the cost to develop, install, and verify the installation, it is expected that this system will be amortized within three years of deployment.

\section{ACKNOWLEDGEMENT}

This work was supported by the U.S. Department of Energy, SO-13, Office of Policy Integration and Technology Support, under contracts DE-AC05-00OR22725 (Oak Ridge National Laboratory) and DE-AC05-00OR22800 (Y-12 National Security Complex).

\section{REFERENCES}

[1] E. Hergert, Hamamatsu Corp., personal communication, February 1, 2002.

[2] M. J. Berger, J. H. Hubbell, S. M. Seltzer, J. S. Coursey and D. S. Zucker, "XCOM: Photon Cross Sections Database (version 1.2)", Available: http://physics.nist.gov/xcom, National Institute of Standards and Technology, Gaithersburg, MD.

[3] Hamamatsu Corp., S3590 Series specification sheet, catalog number KPIN1052E02, January 2002.

[4] E. J. Kennedy, “Operational Amplifier Circuits”, International Thomson Publishing, 1988, p. 399. 